\title{
Regulators of the hypoxic response: a growing family
}
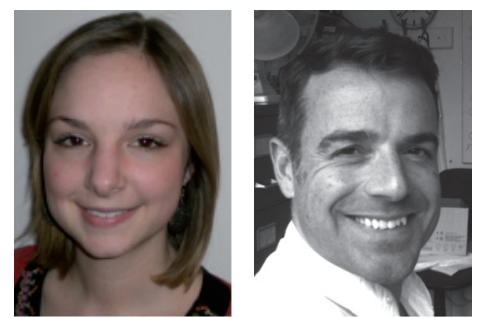

"Deregulation of the hypoxic response is widely accepted as a key step in cellular transformation and subsequent tumorigenesis and metastasis."

Katherine S Bridge' \& Tyson V Sharp*

'School of Biomedical Sciences, University of Nottingham, Queen's Medical Centre, NG7 2UH, UK *Author for correspondence: Barts Cancer Institute, Queen Mary University of London, Charterhouse Square, London ECTM 6BQ. UK=t.sharp@qmul.ac.uk

In recent years there has been a significant increase in the identification of previously unrecognized protein regulators of the hypoxic response. In this editorial, we highlight this growing family, including our recent identification of the Ajuba family of LIM domain-containing proteins. Such proteins are now considered key regulators of the hypoxic response, an adaptive cellular signaling pathway that regulates the cellular and physiological response to changes in oxygen levels by controlling degradation and transcriptional activity of hypoxia-inducible transcription factors (HIFs). Deregulation of the hypoxic response is widely accepted as a key step in cellular transformation and subsequent tumorigenesis and metastasis.

\section{Responding to hypoxia}

In order to survive in a given environment, optimum levels of oxygen are required throughout cells and tissues, as defined by cellular function and tissue type. Oxygen homeostasis is therefore tightly regulated in metazoans to ensure that tissues are sufficiently perfused. While partial pressure of oxygen $\left(\mathrm{pO}_{2}\right)$ within the human body ranges from $150 \mathrm{mmHg}$ in the upper airway to an average of $40 \mathrm{mmHg}$ in most organs, each tissue type has a basal requirement for oxygen under which essential cellular processes such as mitochondrial respiration, protein synthesis and ion transport become impaired [1-4]. Therefore, rapid reaction and adaptation of a cell or tissue to low $\mathrm{pO}_{2}$ (hypoxia) can enable it to remain viable, thus reducing potential damage to the organism.

The hypoxic response signaling pathway is activated by HIFs, initiating a cascade of transcriptional events that result in an increase in levels of proteins such as VEGF and EPO $[5,6]$. These and other hypoxia-responsive gene products promote survival in low $\mathrm{O}_{2}$ conditions through increasing oxygen delivery to the cell and adapting intracellular regulatory pathways. Regulation of the hypoxic response is a tightly coordinated process. HIF is a heterodimer comprised of HIF- $\alpha$ and HIF- $\beta$ subunits, of which HIF- $\alpha$ is degraded in an oxygen-dependent process whereby it is hydroxylated with available molecular oxygen by PHD1-3 proteins, and once hydroxylated engaged by the VHL protein, the recognition component of an E3 ubiquitin ligase [7-10]. HIF- $\alpha$ is subsequently ubiquitylated and degraded. The hypoxic response is frequently deregulated in transformed cells due to mutation or deletion of key hypoxic regulatory genes such as VHL and EGLN1 (PHD2), resulting in irregular activation of hypoxia-responsive genes under nonhypoxic conditions.

\section{The hypoxic paradox}

Within the hypoxic response exists a fundamental paradox of how its ubiquitous nature can be translated into specificity of signaling across many different tissue types and transcriptional targets. A degree of specificity is enabled by the isoforms of key proteins within the hypoxic response, including the PHD, VHL, and HIF proteins. The three PHD isoforms (PHD1-3) have different expression across tissue types, HIF- $\alpha$ hydroxylation site specificities and sensitivities to inhibition at low $\mathrm{pO}_{2}[11,12]$. A further hydroxylase enzyme FIH catalyzes the asparaginyl hydroxylation of HIF- $\alpha$ to inhibit transcriptional coactivator p300 recruitment [13]. Three HIF- $\alpha$ isoforms exist; HIF- $1 \alpha$ and HIF-2 $\alpha$ have distinct as well as overlapping tissue expression and transcriptional targets, while HIF-3 $\alpha$ is localized to the cytoplasm and dimerizes with HIF- $1 \alpha / 2 \alpha$ to inhibit their nuclear localization and transcriptional activities [14-16]. VHL has p19 and p30 isoforms, which although reported to exert the same effect upon HIF- $\alpha$, have some

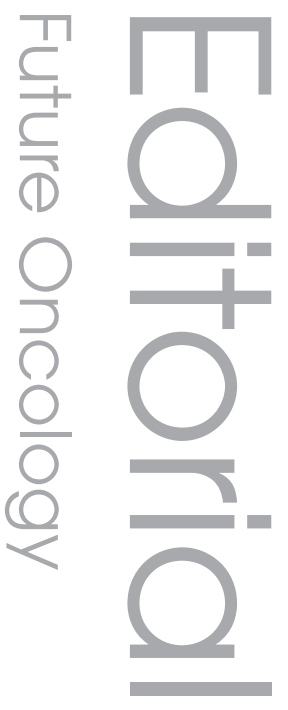

Keywords

- HIF $\approx$ hypoxia $=$ hypoxic regulator proteins $=$ hypoxic response $=$ LIMDI

\section{Future $\because$ Medicine part of}


distinct binding partners and functions $[17,18]$. Therefore, with such combinations we begin to see how different permutations of the hydroxylase enzymes, VHL isoforms and HIFs across different tissue types and under differing $\mathrm{pO}_{2}$ can begin to confer complexity within this signaling pathway at the level of HIF stability within the cytoplasm.

\section{Regulators of the hypoxic response signaling pathway}

There is now a increasing group of regulatory proteins of the hypoxic response response that, in addition to PHD and VHL proteins (or in combination with them) reveal additional layers of multifaceted regulation within the HIF- $\alpha$ degradation pathway that may enable further understanding of the long-standing hypoxic paradox discussed above. These proteins include Siah1/2, which act to stabilize HIF-1 $\alpha$ in hypoxia by enhancing degradation of PHD1 and 3 [19]. A number of groups have reported that SUMOylation of HIF-1 $\alpha$ can both positively and negatively affect its stability and transcriptional activity $[20,21]$. Some HIF-1 $\alpha$ regulators function in an oxygen-independent manner, including the competitive binding of RACK1 or HSP90 to the PAS-A domain of HIF-1 $\alpha$, resulting in its degradation via Elongin C/CUL-2/RBX1 or stabilization, respectively [22]. A further group of regulators exists that interact with HIF-1 $\alpha$, VHL and Elongin $\mathrm{C}$ to enhance ubiquitination of prolyl-hydroxylated HIF-1 $\alpha$, including SSAT2 and MCM7 [23,24].

\section{The LAW family of HIF regulators}

We have recently identified the LIM domaincontaining proteins LIMD1, Ajuba and WTIP (LAW) as a unique family of regulators of the hypoxic response [25]. Unlike other regulators identified to date, the LAW proteins are able to interact simultaneously with PHDs and VHLs in a multimeric complex. This enables optimum HIF-1 $\alpha$ degradation efficiency due to the increased proximity of the hydroxylation and ubiquitination enzymes compared with LAW-independent PHD/VHL degradation. The identification of LAW gives further insight into the ability of hypoxic regulatory proteins to confer specificity within the hypoxic response. Our recent discoveries demonstrated that LAW LIM domain family members are able to interact with one or more of the PHD and VHL isoforms in vivo. LIMD1 is able to bind to PHD1, 2 and 3 and simultaneously interact with the $\mathrm{p} 30$ isoform of VHL, while Ajuba and WTIP interact with both p19 and p30 VHL isoforms, but cannot bind to PHD2.

"...it is essential that the molecular mechanisms of regulatory proteins of the hypoxic response remain an important focus in molecular oncology research, as

they hold the potential to enhance targeting and improve treatment efficacy of HIF-induced cancers."

The existence of these protein complex variants raises interesting questions about their function; for example, would a PHD1-WTIP-p19 VHL complex confer a different effect upon HIF-1 $\alpha$ degradation than a PHD2-LIMD1-p30 VHL complex? It is possible that these complexes are cell-type specific, or that they enable specificity for PHD substrate recognition (HIF-1/2 $\alpha$ ) and hence specificity for inhibition of HIF transcriptional targets. Our study demonstrated that depletion of LIMD1 using RNAi resulted in enhanced transcription of a number of HIF target genes. Interestingly, the effect of LIMD1 depletion upon BNIP3 (BCL2/adenovirus E1B $19 \mathrm{kDa}$ interacting protein 3) and other HIF target genes was dependent on hypoxic conditions, increased transcription of JMJDIA and ALDOC was observed irrespective of $\mathrm{pO}_{2}$. This indicates that LIMD1 may have gene-specific or $\mathrm{O}_{2}$ tension-specific responses, and hence its role in the degradation of HIF-1 $\alpha$ may enable inhibition of specific HIF target genes.

\section{Targeting the regulator proteins of HIFs may enhance specificity in cancer treatments}

The increasing identification of novel regulators of the hypoxic response may represent a possible explanation of how the simple biochemical or kinetic properties of the hydroxylase-ubiquitin ligase couple may be adapted to provide tissue specificity, length of response and specification of target genes necessary for physiological oxygen homeostasis, and may also provide us with increasingly precise targets for drug development and cancer treatment. A substantial number of antitumorigenic HIF-1-inhibiting drugs have been identified, including the antioxidant $N$-acetyl cysteine, which enhances HIF-1 $\alpha$ degradation via the PHD2-VHL pathway, inhibiting lymphomagenesis $[26,27]$. It is therefore conceivable that such cancer therapeutics could also target the precise interfaces within hypoxic regulator protein-PHD-VHL complexes. This could potentially enhance HIF-1 $\alpha$ degradation in a manner 
specific for both the tumor tissue type and, if these complexes can in fact render specificity for inhibiting precise HIF target genes, even the genes that are upregulated in the tumor. As such, it is essential that the molecular mechanisms of regulatory proteins of the hypoxic response remain an important focus in molecular oncology research, as they hold the potential to enhance targeting and improve treatment efficacy of HIF-induced cancers.

\section{Acknowledgement}

The authors would like to thank DE Foxler for critically reading this Editorial.

\section{Financial \& competing interests disclosure \\ KS Bridge is supported by funding from a Biotechnology and Biological Sciences Research Council (BBSRC) \\ Doctorate Training Award. TV Sharp's research group is funded by the BBSRC (BB/I007571/1 and BB/J010901/1) and Cancer Research UK(C8932/A12733). The authors \\ have no other relevant affliations or financial involve- ment with any organization or entity with a financial interest in or financial conflict with the subject matter or materials discussed in the manuscript apart from those disclosed. \\ No writing assistance was utilized in the production of this manuscript.}

\section{References}

1. Heerlein K, Schulze A, Hotz L, Bartsch P, Mairbaurl H. Hypoxia decreases cellular ATP demand and inhibits mitochondrial respiration of a549 cells. Am. J. Respir. Cell Mol. Biol. 32(1), 44-51 (2005).

2. Connolly E, Braunstein S, Formenti S, Schneider RJ. Hypoxia inhibits protein synthesis through a 4E-BP1 and elongation factor 2 kinase pathway controlled by mTOR and uncoupled in breast cancer cells. Mol. Cell Biol. 26(10), 3955-3965 (2006).

3. Taylor CT, Lisco SJ, Awtrey CS, Colgan SP. Hypoxia inhibits cyclic nucleotidestimulated epithelial ion transport: role for nucleotide cyclases as oxygen sensors. J. Pharmacol. Exp. Ther. 284(2), 568-575 (1998).

4. Ivanovic Z. Hypoxia or in situ normoxia: the stem cell paradigm. J. Cell Physiol. 219(2), 271-275 (2009).

5. Levy AP, Levy NS, Wegner S, Goldberg MA. Transcriptional regulation of the rat vascular endothelial growth factor gene by hypoxia. J. Biol. Chem. 270, 13333-13340 (1995).

6. Semenza GL, Wang GL. A nuclear factor induced by hypoxia via de novo protein synthesis binds to the human erythropoietin gene enhancer at a site required for transcriptional activation. Mol. Cell Biol. 12(12), 5447-5454 (1992).

7. Yu F, White SB, Zhao Q, Lee FS. HIF-1alpha binding to VHL is regulated by stimulussensitive proline hydroxylation. Proc. Natl Acad. Sci. USA. 98(17), 9630-9635 (2001).

8. Jaakkola P, Mole DR, Tian YM et al. Targeting of HIF-alpha to the von Hippel-Lindau ubiquitylation complex by $\mathrm{O}_{2}$-regulated prolyl hydroxylation. Science 292(5516), 468-472 (2001).

9. Ivan M, Kondo K, Yang $\mathrm{H}$ et al. HIFalpha targeted for VHL-mediated destruction by proline hydroxylation: implications for $\mathrm{O}_{2}$ sensing. Science 292 (5516), 464-468 (2001).

10. Maxwell PH, Wiesener MS, Chang GW et al. The tumour suppressor protein VHL targets hypoxia-inducible factors for oxygendependent proteolysis. Nature 399(6733), 271-275 (1999).

11. Jokilehto T, Jaakkola PM. The role of HIF prolyl hydroxylases in tumour growth. J. Cell. Mol. Med. 14(4), 758-770 (2010).

12. Tian YM, Yeoh KK, Lee MK et al. Differential sensitivity of hypoxia inducible factor hydroxylation sites to hypoxia and hydroxylase inhibitors. J. Biol. Chem. 286(15), 13041-13051 (2011).

13. Lando D, Peet DJ, Whelan DA, Gorman JJ, Whitelaw ML. Asparagine hydroxylation of the HIF transactivation domain a hypoxic switch. Science 295(5556), 858-861 (2002).

14. Hu CJ, Wang LY, Chodosh LA, Keith B, Simon MC. Differential roles of hypoxiainducible factor 1alpha (HIF-1alpha) and HIF-2alpha in hypoxic gene regulation. Mol. Cell Biol. 23(24), 9361-9374 (2003).

15. Holmquist-Mengelbier L, Fredlund E, Lofstedt T et al. Recruitment of HIF-1alpha and HIF-2alpha to common target genes is differentially regulated in neuroblastoma: HIF-2alpha promotes an aggressive phenotype. Cancer Cell 10 (5), 413-423 (2006).

16. Heikkilä M, Pasanen A, Kivirikko KI, Myllyharju J. Roles of the human hypoxiainducible factor (HIF)-3alpha variants in the hypoxia response. Cell. Mol. Life Sci. 68(23), 3885-3901 (2011).

17. Cockman ME, Masson N, Mole DR et al. Hypoxia inducible factor-alpha binding and ubiquitylation by the von Hippel-Lindau tumor suppressor protein. J. Biol. Chem. 275(33), 25733-25741 (2000).

18. Lai Y, Song M, Hakala K, Weintraub ST, Shiio Y. Proteomic dissection of the von
Hippel-Lindau (VHL) interactome. J. Proteome Res. 10 (11), 5175-5182 (2011).

19. Nakayama K, Frew IJ, Hagensen M et al. Siah2 regulates stability of prolylhydroxylases, controls HIFlalpha abundance, and modulates physiological responses to hypoxia. Cell 117(7), 941-952 (2004).

20. Cheng J, Kang X, Zhang S, Yeh ET. SUMO-specific protease 1 is essential for stabilization of HIF1alpha during hypoxia. Cell 131(3), 584-595 (2007).

21. Carbia-Nagashima A, Gerez J, Perez-Castro C et al. RSUME, a small RWD-containing protein, enhances SUMO conjugation and stabilizes HIF-1alpha during hypoxia. Cell 131(2), 309-323 (2007).

22. Liu YV, Semenza GL. RACK1 vs. HSP90: competition for HIF-1 alpha degradation vs. stabilization. Cell Cycle 6(6), 656-659 (2007).

23. Baek JH, Liu YV, McDonald KR et al. Spermidine/spermine-N1-acetyltransferase 2 is an essential component of the ubiquitin ligase complex that regulates hypoxiainducible factor 1alpha. J. Biol. Chem. 282(32), 23572-23580 (2007).

24. Hubbi ME, Luo W, Baek JH, Semenza GL. $\mathrm{MCM}$ proteins are negative regulators of hypoxia-inducible factor 1. Mol. Cell 42(5), 700-712 (2011).

25. Foxler DE, Bridge KS, James V et al. The LIMD1 protein bridges an association between the prolyl hydroxylases and VHL to repress HIF-1 activity. Nat. Cell Biol. 14(2), 201-208 (2012).

26. Semenza GL. Hypoxia-inducible factors: mediators of cancer progression and targets for cancer therapy. Trends Pharmacol. Sci. 33(4), 207-214 (2012).

27. Gao P, Zhang H, Dinavahi R et al. HIFdependent antitumorigenic effect of antioxidants in vivo. Cancer Cell 12(3), 230-238 (2007). 